\title{
Odd-Frequency Density Waves: Non-Fermi-Liquid Metals with an Order Parameter
}

\author{
Eugene Pivovarov \\ Department of Physics, Mail Code 103-33, Caltech, Pasadena, CA 91125 \\ Chetan Nayak \\ Department of Physics and Astronomy, University of California, Los Angeles, CA 90095-1547
}

(November 20, 2018)

\begin{abstract}
We consider states with a charge- or spin-density wave order parameter which is odd in frequency, so that the order parameter vanishes at zero frequency and there is a conventional Fermi surface. Such states break translational symmetry and, therefore, are not conventional Fermi liquids. In the odd-frequency spin-density wave case, there are Goldstone bosons and the low-energy spectrum is manifestly different from that of a Fermi liquid. We discuss a simple model which gives rise to such ordered states. The frequency-dependence of the gap leads to an unusual temperature dependence for various thermodynamic and transport properties, notably the resistivity.

PACS numbers: 71.10.Hf, 71.45.Lr, 72.15.-v
\end{abstract}

\section{INTRODUCTION}

The Fermi liquid is a metallic state which is adiabatically continuable to a non-interacting electron gas. Its low-energy excitations are in one-to-one correspondence with those of the non-interacting system. Many attempts to uncover non-Fermi-liquid metallic behavior in strongly-correlated electron systems have focused on enhanced scattering mechanisms which might lead to anomalous behavior in the electron Green function. In this paper, we attempt a different tack and focus on metallic states with a conventional Fermi surface which can be distinguished from Fermi liquids by an order $p a-$ rameter. In order to preserve the Fermi surface, the order parameter is taken to be odd in frequency [1]. When the order parameter breaks a continuous symmetry, the lowenergy spectrum must also include Goldstone modes. As a result, odd-frequency ordering is a mechanism by which the set of low-energy excitations can be expanded from those of a Fermi liquid (so that they are no longer in oneto-one correspondence with those of a free Fermi gas), leading to a manifestly non-Fermi liquid state.

A number of authors have considered superconducting states with order parameters $\left\langle c_{\alpha}(k, \omega) c_{\beta}(-k,-\omega)\right\rangle$ which are odd in frequency [2 [7]. The energetic advantage of such a state is that it would enable the electrons to avoid instantaneous Coulomb repulsion while still benefitting from pairing. Some exactly soluble one-dimensional models, such as the one-dimensional Kondo lattice, have a tendency towards such ordering 88.97. However, there are claims that the simplest models of odd-frequency superconducting states suffer from pathologies which make them unstable [7, 10].

In this paper, we consider the analogous states in the particle-hole channels. We find that simple models of odd-frequency density wave states do not suffer from any pathologies (and suspect that this holds for the corresponding superconducting states, as well). Oddfrequency states can exhibit a number of interesting nonFermi liquid properties including the Goldstone modes mentioned above, a range of states above the Fermi surface with finite lifetimes even in the limit of vanishing frequency and temperature $\epsilon, T \rightarrow 0$, and a non-meanfield-like temperature-dependent order parameter.

However, the question of their detection is non-trivial and cuts to the heart of attempts to experimentally distinguish non-Fermi liquids from Fermi liquids. While an odd-frequency superconducting state is, first and foremost, a superconducting state, which would be identified by its vanishing resistivity, Meissner effect, etc., an odd-frequency density wave state can masquerade as a Fermi liquid since the order parameter vanishes at zero frequency. There will be signatures in thermodynamic and transport measurements, but they can easily be mistakenly attributed to other effects, as we discuss below.

\section{ORDER PARAMETERS AND SYMMETRIES}

An odd-frequency charge-density wave state is defined by the anomalous correlation function

$$
\left\langle c^{\alpha \dagger}\left(k, \epsilon_{n}\right) c_{\alpha}\left(k+Q, \epsilon_{n}\right)\right\rangle=F\left(k, \epsilon_{n}\right),
$$

where $F\left(k, \epsilon_{n}\right)$ is an odd function of frequency,

$$
F\left(k,-\epsilon_{n}\right)=-F\left(k, \epsilon_{n}\right) .
$$

To find an equal-time correlation function which serves as an order-parameter, we Fourier transform (1):

$$
\left\langle T_{\tau}\left(c^{\alpha \dagger}(k, \tau) c_{\alpha}(k+Q, 0)\right)\right\rangle=\tilde{F}(k, \tau),
$$

where $\tilde{F}(k, \tau)$ is imaginary and odd in $\tau$. Hence, we can use the time-derivative as an order parameter:

$$
\left\langle T_{\tau}\left(\partial_{\tau} c^{\alpha \dagger}(k, \tau) c_{\alpha}(k+Q, 0)\right)\right\rangle_{\tau=0}=\partial_{\tau} \tilde{F}(k, 0) .
$$


The state defined by these order parameters, (1) or (4), breaks translational symmetry. Time-reversal symmetry is not broken. This is most easily seen by considering (3). Taking the complex conjugate of both sides of (3) gives

$$
\begin{aligned}
\left\langle T_{\tau}\left(c^{\alpha \dagger}(k+Q, 0) c_{\alpha}(k, \tau)\right)\right\rangle & =(\tilde{F}(k, \tau))^{*} \\
& =-\tilde{F}(k, \tau) .
\end{aligned}
$$

Meanwhile, (3) is transformed under time-reversal, $\mathcal{T}$, into:

$$
\begin{aligned}
\left\langle T_{\tau}\right. & \left.\left(\mathcal{T}\left(c^{\alpha \dagger}(k, \tau) c_{\alpha}(k+Q, 0)\right)\right)\right\rangle \\
& =\left\langle T_{\tau}\left(c^{\alpha \dagger}(k+Q, 0) c_{\alpha}(k,-\tau)\right)\right\rangle \\
& =-\tilde{F}(k,-\tau) \\
& =\tilde{F}(k, \tau) \\
& =\left\langle T_{\tau}\left(c^{\alpha \dagger}(k, \tau) c_{\alpha}(k+Q, 0)\right)\right\rangle,
\end{aligned}
$$

and, hence, the order parameter does not break timereversal symmetry. In going from the first equality to the second, we have used (5).

An odd-frequency spin-density wave is defined by

$$
\left\langle c^{\alpha \dagger}\left(k, \epsilon_{n}\right) c_{\beta}\left(k+Q, \epsilon_{n}\right)\right\rangle=\vec{n} \cdot \vec{\sigma}_{\beta}^{\alpha} F\left(k, \epsilon_{n}\right),
$$

where $F\left(k, \epsilon_{n}\right)$ is again an odd function of frequency and $\vec{n}$ is the direction chosen spontaneously by the ordered state. This state breaks translational symmetry and spinrotational symmetry, which is broken to the $U(1)$ subgroup of rotations about $\vec{n}$. Again, time-reversal is preserved. Such a state will exhibit a non-zero expectations value and anomalous correlations of the spin nematic order parameter, $S_{i} S_{j}-\delta_{i j} S^{2} / 3$, such as those discussed for spin-only models in 11 .

\section{MODEL INTERACTION}

We now consider a simple two-dimensional model which admits an odd-frequency charge-density-wave state at the mean-field level. The model contains a nonsingular four-fermion interaction which can be generated by the exchange of phonons or some gapped electronic collective mode. For simplicity, we focus on the chargedensity-wave case; the spin-density-wave is analogous.

We consider an effective action which consists of a kinetic term

$$
S_{0}=\int d \tau \int \frac{d^{2} k}{(2 \pi)^{2}} c^{\alpha \dagger}(k, \tau)\left(\partial_{\tau}-(\epsilon(k)-\mu)\right) c_{\alpha}(k, \tau)
$$

and an interaction term

$$
\begin{aligned}
S_{\mathrm{int}}=\frac{1}{\Omega_{c}^{2}} & \int d \tau \int_{k, k^{\prime}}\left[c^{\alpha \dagger}(k+Q) \partial_{\tau} c_{\alpha}(k)\right]_{c} V_{k k^{\prime}} \\
& \times\left[c^{\beta \dagger}\left(k^{\prime}\right) \partial_{\tau} c_{\beta}\left(k^{\prime}+Q\right)\right]_{c}
\end{aligned}
$$

To avoid clutter, the $\int_{k, k^{\prime}}$ is used as shorthand for the integrals over $k, k^{\prime}$. The subscript $c$ indicates that the terms in brackets are actually defined with a frequency cutoff, $\Omega_{c}$ :

$$
\begin{aligned}
& {\left[c^{\alpha \dagger}(k+Q) \partial_{\tau} c_{\alpha}(k)\right]_{c} \equiv} \\
& \quad T \sum_{\left|\epsilon_{n}\right|<\Omega_{c}} i \epsilon_{n} c^{\alpha \dagger}\left(k+Q, \epsilon_{n}\right) c_{\alpha}\left(k, \epsilon_{n}\right) .
\end{aligned}
$$

For simplicity, we take $V_{k k^{\prime}}$ independent of $k, k^{\prime}, V_{k k^{\prime}}=$ $\lambda$. For simplicity, we also take $Q=(\pi, \pi)$ and $\epsilon(k)=$ $-2 t\left(\cos k_{x}+\cos k_{y}\right)$, corresponding to commensurate order for a system of electrons on a square lattice with nearest-neighbor hopping. The generalization to incommensurate order and other band structures is straightforward.

The interaction term $S_{\text {int }}$ is long-ranged in precisely the same way as the BCS reduced interaction. A more realistic short-ranged interaction would be of the form

$$
\begin{aligned}
\tilde{S}_{\mathrm{int}}=\int_{k, k^{\prime}, q} & {\left[c^{\alpha \dagger}(k+q) \partial_{\tau} c_{\alpha}(k)\right]_{c} V_{k k^{\prime}}^{q} } \\
\times & {\left[c^{\beta \dagger}\left(k^{\prime}\right) \partial_{\tau} c_{\beta}\left(k^{\prime}+q\right)\right]_{c}, }
\end{aligned}
$$

which includes (9) as one term in the sum over $q$. Such an interaction could arise from the diagram of figure 1 if the collective mode has a propagator of the form

$$
\lambda \frac{\Omega_{c}^{2}}{\Omega_{c}^{2}+v^{2}\left(k-k^{\prime}\right)^{2}+\left(\epsilon_{n}-\epsilon_{n^{\prime}}\right)^{2}} .
$$

If $v$ is small, then we can expand the collective mode propagator to obtain $V_{k k^{\prime}}=\lambda$, for $\left|\epsilon_{n}\right|,\left|\epsilon_{n^{\prime}}\right| \leq \Omega_{c}$. Other terms will also be generated which could drive the formation of even-frequency order, but they appear to be weaker.

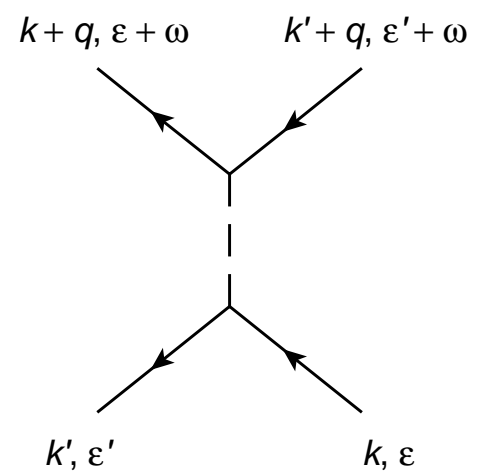

FIG. 1. A diagram which can lead to an interaction favorable for odd-frequency density-wave ordering. The dashed line represents a collective mode which mediates the interaction. 
We assume an order parameter of the form:

$$
\alpha \equiv \frac{\lambda}{\Omega_{c}^{2}} \int \frac{d^{2} k}{(2 \pi)^{2}}\left\langle c^{\alpha \dagger}(k+Q) i \partial_{\tau} c_{\alpha}(k)\right\rangle_{c} .
$$

According to our previous observations, $\alpha$ is real. Then the mean-field action takes the form

$$
\begin{aligned}
S_{0}= & T \sum_{n} \int_{k} c^{\alpha \dagger}\left(k, \epsilon_{n}\right)\left(i \epsilon_{n}-(\epsilon(k)-\mu)\right) c_{\alpha}\left(k, \epsilon_{n}\right) \\
& -T \sum_{n} \int \frac{d^{2} k}{(2 \pi)^{2}} \alpha \epsilon_{n} c^{\alpha \dagger}\left(k, \epsilon_{n}\right) c_{\alpha}\left(k+Q, \epsilon_{n}\right) .
\end{aligned}
$$

The equation of motion following from the mean-field action is:

$$
\left(i \epsilon_{n}-\epsilon(k)+\mu\right) c_{\alpha}\left(k, \epsilon_{n}\right)-\alpha \epsilon_{n} c_{\alpha}\left(k+Q, \epsilon_{n}\right)=0 .
$$

We multiply the equation of motion by $c_{\alpha}^{\dagger}\left(k, \epsilon_{n}\right)$ and take the imaginary time-ordered expectation value. We see that the ordinary and anomalous Green functions satisfy the equation:

$$
\left(i \epsilon_{n}-\epsilon(k)+\mu\right) G\left(k, \epsilon_{n}\right)-\alpha \epsilon_{n} F\left(k, \epsilon_{n}\right)=1 .
$$

The right-hand-side results from the time-derivative (i.e., $\left.i \epsilon_{n}\right)$ acting on the time-ordering symbol [12]. If we make the replacement $k \rightarrow k+Q$ in (15), then we can derive a second equation in the same way,

$$
\left(i \epsilon_{n}+\epsilon(k)+\mu\right) F\left(k, \epsilon_{n}\right)-\alpha \epsilon_{n} G\left(k, \epsilon_{n}\right)=0,
$$

and the Green function and anomalous Green function for $\left|\epsilon_{n}\right| \leq \Omega_{c}$ take the form:

$$
\begin{aligned}
& G\left(k, \epsilon_{n}\right)=\frac{i \epsilon_{n}+\mu+\epsilon(k)}{\left(i \epsilon_{n}+\mu\right)^{2}-(\epsilon(k))^{2}-\alpha^{2} \epsilon_{n}^{2}}, \\
& F\left(k, \epsilon_{n}\right)=\frac{\alpha \epsilon_{n}}{\left(i \epsilon_{n}+\mu\right)^{2}-(\epsilon(k))^{2}-\alpha^{2} \epsilon_{n}^{2}} .
\end{aligned}
$$

One might naively think that equation (17) could be obtained by inspection from (16) by replacing $\Delta\left(\epsilon_{n}\right)$ by $\Delta^{*}\left(-\epsilon_{n}\right)$ as one usually does in the case of an evenfrequency gap. In this case, this would amount to the replacement of $\alpha \epsilon_{n}$ by $-\alpha \epsilon_{n}$, as was done in Ref. [7, 10]. However, in the case of an odd-frequency gap, this simple substitution only works for real frequencies. Since the gap is linear in frequency and the squared modulus of the gap appears in the Green functions, the Green functions are no longer analytic in the frequency. As a result, the analytic continuation from real to Matsubara frequencies is subtle. The safe route is to derive both (16) and (17) directly from the mean-field action, as we have done. The naive, incorrect form of (17) would lead to a negative superfluid density in the case of odd-gap superconductors. On the other hand, the correct analogue of (17) for a superconducting action induced by disorder was used in Ref. [6].
For $\left|\epsilon_{n}\right|>\Omega_{c}, F$ vanishes and $G$ returns to its normal state form. In principle, we should also allow for a quasiparticle renormalization $Z$ resulting from the interaction, but this does not qualitatively modify our results, so we drop this correction for simplicity.

From these Green functions, we see that, at $\mu=0$, the odd-frequency charge-density-wave order parameter modifies the quasiparticle spectrum to:

$$
E(k) \equiv \frac{\epsilon(k)}{\sqrt{1+\alpha^{2}}},
$$

i.e., it renormalizes the effective mass. For $\mu \neq 0$, the effect is more complicated. As a result of the odd-frequency charge-density-wave, $\epsilon(k)-\mu$ is replaced with

$$
E(k) \equiv \frac{-\mu \pm \sqrt{\left(1+\alpha^{2}\right) \epsilon^{2}(k)-\alpha^{2} \mu^{2}}}{1+\alpha^{2}} .
$$

From (20), we see that the Fermi surface is unmoved, i.e., $E(k)=0$ when $\epsilon(k)=\mu$, as we expect, since the order parameter vanishes at zero frequency.

Furthermore, there is a range of $k$ values above the Fermi surface, $|\epsilon(k)|<\left|\alpha \mu / \sqrt{1+\alpha^{2}}\right|$, where $E(k)$ has an imaginary part, so that quasiparticles in this region have a finite lifetime. However, these states have zero occupation number, as the corresponding poles in the Green functions turn out to be lying outside of the integration contour. This gives rise to a possibility of the ground state in which some of the quasiparticles are in levels which are in disconnected from the rest of the Fermi sea.

\section{GAP EQUATION}

We must now impose a self-consistency condition on $F\left(k, \epsilon_{n}\right)$, which is the gap equation. We will also impose a condition on the particle number, thereby implicitly fixing the chemical potential. These conditions read:

$$
\begin{aligned}
-\frac{\lambda}{\Omega_{c}^{2}} 2 T \sum_{n}^{\prime} \int_{k} \frac{\epsilon_{n}^{2}}{\left(i \epsilon_{n}+\mu\right)^{2}-(\epsilon(k))^{2}-\alpha^{2} \epsilon_{n}^{2}} & =1, \\
T \sum_{n}^{\prime} \int_{k} \frac{i \epsilon_{n}+\mu+\epsilon(k)}{\left(i \epsilon_{n}+\mu\right)^{2}-(\epsilon(k))^{2}-\alpha^{2} \epsilon_{n}^{2}} & =n .
\end{aligned}
$$

For simplicity, we consider the case of half-filling, $n=1$. We have repeated our calculations at non-zero doping and found similar results.

The prime on the Matsubara summations in 21a), (21b) indicate that they are done with $\alpha=0$ for $\left|\epsilon_{n}\right|>\Omega_{c}$ and $\alpha \neq 0$ only for $\left|\epsilon_{n}\right| \leq \Omega_{c}$. A more realistic model replaces the interaction with one that has a "smooth" cutoff $s_{\eta}\left(\epsilon_{n}\right) s_{\eta}\left(\epsilon_{n^{\prime}}\right)$ and $\alpha$ by $\alpha s_{\eta}\left(\epsilon_{n}\right)$, with $s_{\eta}(\epsilon)=1$ for $\epsilon \ll \Omega_{c}$ and $s_{\eta}(\epsilon)=0$ for $\epsilon \gg \Omega_{c}$. We can vary $\eta$ between the limit $\eta \rightarrow \infty$, which corresponds to a 
sharp cutoff, and $\eta \rightarrow 0$ which corresponds to the absence of a cutoff. For computational simplicity, we take $s_{\eta}(\epsilon)=n_{F}\left(|\epsilon|-\Omega_{c} ; \beta=\eta\right)$.

We now discuss the analysis of $(21 a),(21 b$. . Let us first consider the case of a sharp cutoff. The left-hand side of the gap equation vanishes if the temperature is above

$$
T_{c}^{\max }=\frac{\Omega_{c}}{\pi}
$$

$T_{c}^{\max }$ is the highest possible transition temperature for this system. Just below this temperature there is only one pair of terms in the Matsubara sum which is allowed by the cutoff $\Omega_{c}$. As the temperature is decreased, more Matsubara frequencies begin to contribute, resulting in minor steps in the phase diagram.

For large $\lambda, T_{c}=T_{c}^{\max }$. Decreasing $\lambda$, we enter a regime, $\lambda_{c 2}<\lambda<\lambda_{c 1}$, in which the system is in the oddfrequency density-wave phase for an intermediate range of temperatures $T_{c 2}<T<T_{c 1} . \lambda_{c 1}$ is the location of the quantum phase transition at which the odd-frequency density-wave order first appears at zero temperature. It is obtained from (21a) by setting $\mu=0$ and $\alpha=0$ and converting the Matsubara sum into an integral:

$$
\int \frac{d^{2} k}{(2 \pi)^{2}}\left[\Omega_{c}-\epsilon(k) \arctan \frac{\Omega_{c}}{\epsilon(k)}\right]=\frac{\pi \Omega_{c}^{2}}{\lambda_{c 1}} .
$$

The integrand is approximately $\Omega_{c}$ for small $\epsilon(k)$ and $\Omega_{c}^{3} / 3 \epsilon^{2}(k)$ for $|\epsilon(k)| \gg \Omega_{c}$.

As $\lambda$ is further decreased, $T_{c 2}$ increases and finally reaches $T_{c}^{\max }$ at $\lambda_{c 2}$. To find $\lambda_{c 2}$, we again set $\mu=0$ and $\alpha=0$, but now we retain only the pair of terms in the Matsubara sum corresponding to the frequencies $\pm \Omega_{c}$ :

$$
\int \frac{d^{2} k}{(2 \pi)^{2}} \frac{T_{c}^{\max }}{\Omega_{c}^{2}+\epsilon^{2}(k)}=\frac{1}{\lambda_{c 2}} .
$$

Comparing expressions (23) and (24), we find that the latter is larger by a factor of 3 for large $\epsilon(k)$; hence, $\lambda_{c 2}<\lambda_{c 1}$. For $\lambda>\lambda_{c 2}, \alpha$ jumps discontinuously at $T_{c}^{\max }$. Finally, for $0<\lambda<\lambda_{c 2}$, there are no oddfrequency charge-density-wave solutions. As a result, the phase diagram has the shape shown in Fig. 2 (dashed line) with re-entrant transitions for $\lambda_{c 2}<\lambda<\lambda_{c 1}$.

Let us now consider how this picture is modified when we make the cutoff smooth, as it must be in a physical system. The sharpness of the steps which separate the re-entrant transitions depends on the details of the highfrequency cutoff; they disappear in the limit that the cutoff is very smooth. When the cutoff is relatively sharp, more re-entrant transitions are possible, and there will be several temperature regions in which an odd-frequency charge-density wave occurs. However, for a smooth cutoff, there is typically only one such region. The transition at $T_{c}^{\max }$ is replaced with a smooth curve $T_{c 1}(\lambda)$, at which a second-order transition takes place. The corresponding phase boundary is depicted by the solid line in Fig. 2 .

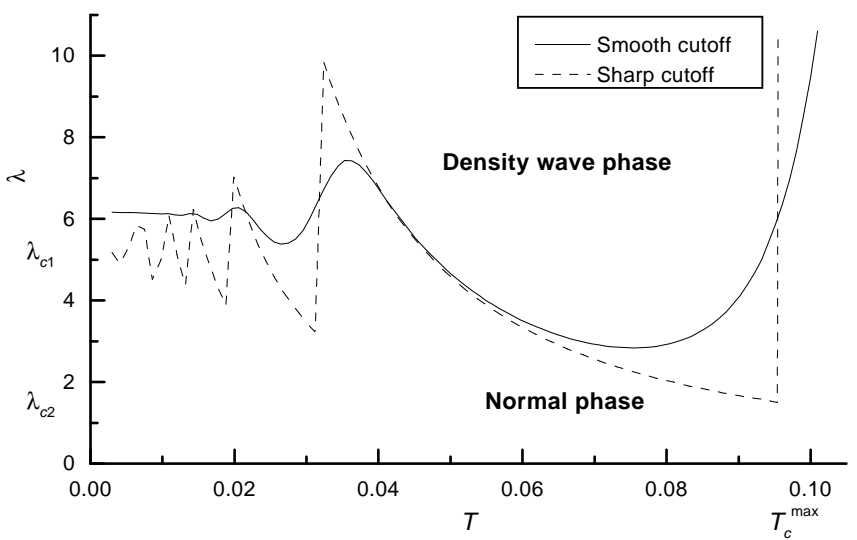

FIG. 2. Phase diagram at half-filling for "smooth" and "sharp" cutoffs at $\Omega=0.3$. The energy scale is set by the width of the band $W=2$.

Below $T_{c 1}(\lambda), \alpha$ increases as shown in Fig. 3. Note that for a smooth cutoff all transitions are of second order, even though the rise of $\alpha$ at $T_{c 1}$ is very steep for large values of $\lambda$ and may give a false impression of a first-order transition. It is also noteworthy that even for large $\lambda$ the order parameter $\alpha$ rapidly attains its maximum as $T$ is decreased below $T_{c}$ and then decreases as $T \rightarrow 0$ to some non-zero asymptotic value. This has a significant impact on experimentally measurable parameters, as we describe in the following section.

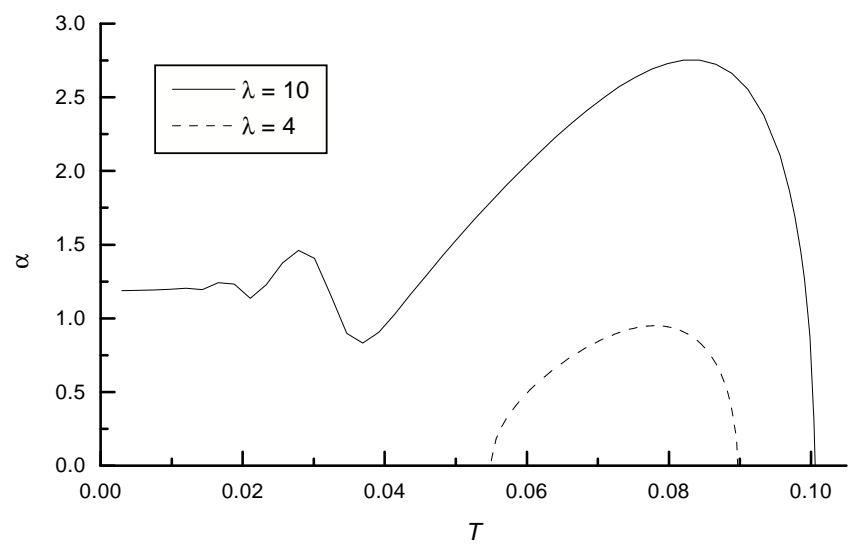

FIG. 3. Order parameter for different values of the interaction strength, $\lambda$, with a "smooth" cutoff at $\Omega=0.3$.

The unusual temperature dependence of the order parameter, which is reflected in the re-entrant phase diagram and (as we will see in the next section) the condensation energy, is a consequence of the frequencydependence of the gap. In an ordinary, frequencyindependent (or weakly-dependent) ordered state, the condensation energy at weak-coupling comes primarily from states near the Fermi energy. At high tempera- 
tures, these states are thermally excited, so there is little condensation energy to be gained, and the order parameter decreases as the temperature is increased. In the case of odd-frequency ordered states, there is very little condensation energy to be gained from the particles near the Fermi surface because their energy is low (and, hence, they interact weakly with the order parameter). As a result, the order parameter and condensation energy decrease as the temperature is decreased.

In fact, there is a second solution to the gap equation in which it is favorable to have a disconnected Fermi sea in which some electrons are excited to a strip in momentum space above the Fermi surface which is diconnected from the rest of the Fermi sea, which is centered about $\mathbf{k}=0$. When this occurs, the occupation number does not increase monotonically with $\epsilon(k)$. However, this solution is higher in energy, so it does not occur.

\section{EXPERIMENTAL SIGNATURES}

At $T_{c}$, there will be the usual thermodynamic signatures of a second order phase transition. The condensation energy associated with this transition is the difference between the free energy of the odd-frequency chargedensity-wave state and corresponding free energy of the normal state at the same temperature. At $\mu=0$ and with a sharp cutoff,

$$
\begin{aligned}
\Delta E(T)= & \frac{\Omega_{c}^{2}}{\lambda} \alpha^{2}+ \\
& 2 T \sum_{n=-n_{c}-1}^{n_{c}} \int_{k} \ln \left(\frac{\epsilon(k)^{2}+\epsilon_{n}^{2}}{\left(1+\alpha^{2}\right) \epsilon_{n}^{2}+\epsilon(k)^{2}}\right),
\end{aligned}
$$

where $n_{c}=\left(\Omega_{c} / \pi T-1\right) / 2$. This equation is obtained by using $\alpha$ as a Hubbard-Stratonovich field to decouple (9), resulting in the first term in (25). The electronic action is then the mean-field action (14), so that the partition function may be evaluated to give the second term in 25. At zero temperature, the integrals may be evaluated analytically:

$$
\begin{aligned}
\Delta E(0)= & \frac{\Omega_{c}^{2}}{\lambda} \alpha^{2}+\frac{2}{\pi} \int_{k} \epsilon(k) \arctan \left(\frac{\Omega_{c}}{\epsilon(k)}\right) \\
& -\frac{2}{\pi} \int_{k} \frac{\epsilon(k)}{\sqrt{1+\alpha^{2}}} \arctan \left(\frac{\Omega_{c} \sqrt{1+\alpha^{2}}}{\epsilon(k)}\right) \\
& +\Omega_{c} \frac{2}{\pi} \int_{k} \ln \left(\frac{\Omega_{c}^{2}+\epsilon^{2}(k)}{\left(1+\alpha^{2}\right) \Omega_{c}^{2}+\epsilon^{2}(k)}\right) .
\end{aligned}
$$

The last term is overwhelmingly negative, as may be seen in various limits (e.g., $\alpha \ll 1$ or $\Omega_{c} \rightarrow \infty$ ). Note that the energetic gain comes not from the terms in the frequency sum (25) with small Matsubara frequency, which actually increase energy, but from the terms near the cutoff
- in a complete reversal of the situation for a frequencyindependent gap 13 .

Again, for a smooth cutoff the Matsubara frequency sum becomes infinite and $\alpha$ should be replaced with $s_{\eta}\left(\epsilon_{n}\right) \alpha$. For smooth cutoff and finite temperature, the condensation energy must be evaluated numerically. The dependence of the condensation energy $\Delta E$ on temperature is shown in Fig. 4 . Unlike in even-frequency phases, where $|\Delta E|$ slowly increases as the system cools down and attains its maximum at zero temperature, in our model it rapidly reaches a maximum and decreases to a constant asymptotic value as $T \rightarrow 0$. As may be seen in figure 4 , the condensation energy is of order of $N_{F} T_{c 1}^{2}$ at the maximum, which is comparable to the maximum condensation energy attained in even-frequency phases.

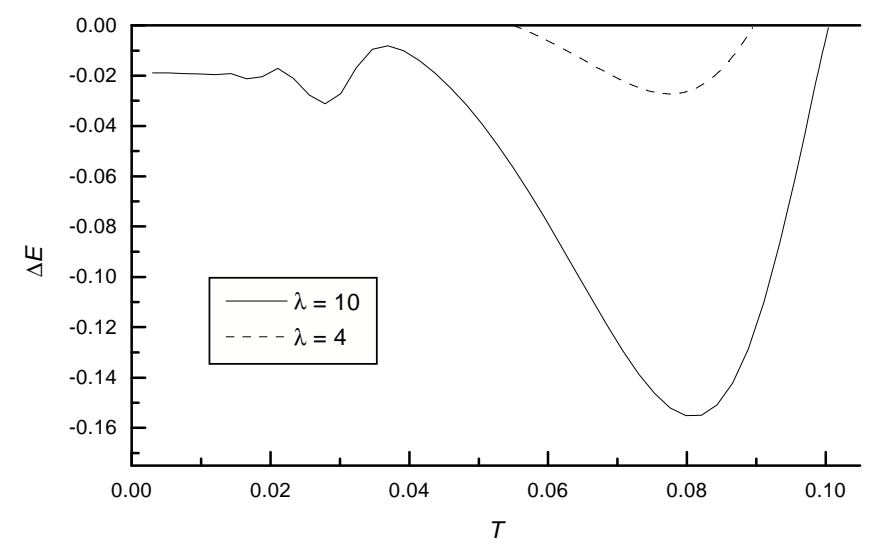

FIG. 4. Condensation energy as a function of temperature in model (9) with a "smooth" cutoff at $\Omega=0.3$.

The DC conductivity can be computed from the Kubo formula. We assume a model in which impurities give rise to a lifetime, $\tau$. The resulting conductivity is given by

$$
\sigma=\frac{1}{1+\alpha^{2}} \int_{k}\left(\frac{\partial \epsilon(k)}{\partial k_{x}}\right)^{2} \frac{\tau}{4 T} \frac{1}{\cosh \left(\frac{E(k)}{2 T}\right)^{2}}
$$

where $E(k)$ is given in (20). For small $\mu$, the modification of the quasiparticle spectrum in the odd-frequency charge-density-wave phase reduces to the rescaling of the electron mass near the Fermi surface, so that the new effective mass is $m^{*}=m \sqrt{1+\alpha^{2}}$. This mass enhancement leads to a noticeable increase of the resistivity, as shown in Fig. 5. Of course, outside of the the region $T_{c 2}(\lambda)<T<T_{c 1}(\lambda)$ the resistivity is that of the normal phase. 


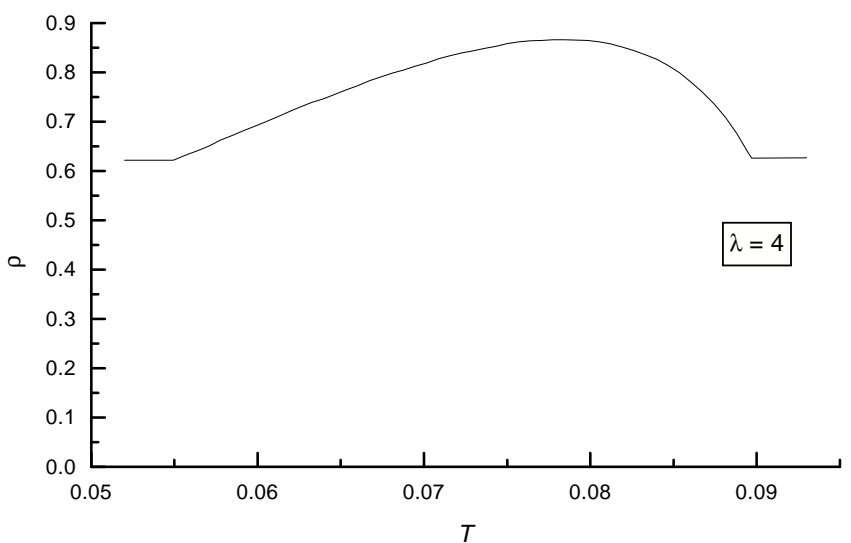

FIG. 5. Resistivity as a function of temperature in the model of Eq. (9) with a "smooth" cutoff at $\Omega=0.3$ and interaction strength $\lambda=4$.

\section{DISCUSSION}

In this work we have considered a model with an oddfrequency charge-density-wave solution. The transition to this state is signaled by a second-order phase transition with a jump in the specific heat. For strong interactions, the model is in an odd-frequency charge-densitywave phase for all temperatures $T<T_{c}$. For moderately weak interactions, the model is in such a phase for an intermediate temperature regime $T_{c 1}<T<T_{c 2}$. There is a quantum phase transition at $\lambda=\lambda_{c 1}$; for weaker interactions, the system is not ordered at $T=0$.

A similar model admits an odd-frequency spin-densitywave ground state. Such a state will have, in addition to its Fermi-liquid-like quasiparticles, Goldstone boson excitations. As a result $S_{i} S_{j}-\delta_{i j} S^{2} / 3$, has a non-zero expectation value, and its correlation functions have Goldstone poles.

The method that we used to derive the Green functions of the odd-frequency density wave can be applied to the analysis of odd-frequency superconductivity as well, with some modification. The corresponding mean field theory should describe a stable state with positive superfluid density.

Odd-frequency charge-density wave order results in mass enhancement. This affects transport properties; in the density-wave state the resistivity is considerably larger than in the normal state. The effect is largest at intermediate temperatures. At low temperatures, the system is either in the normal state (for $\lambda<\lambda_{c 1}$ ) or in an ordered state (for $\lambda \geq \lambda_{c 1}$ ) with some asymptotic value of the order parameter. This type of nonmonotonic resistivity curve has been observed in a number of strongly-correlated electron systems. In layered materials such as the cuprates and ruthenates, it has been observed in $c$-axis transport [14]. In 2DEGs, this type of behavior has been observed in the vicinity of a putative metal-insulator transition [15]. It would be pre- mature to suggest that odd-frequency order is developing in any of these experiments, but it is noteworthy that it does provide a natural explanation of otherwise puzzling behavior.

Odd-frequency density wave ordering is also manifested in thermodynamics. Unlike in even-frequency states, where the condensation energy $|\Delta E|$ is small near the phase transition and reaches a maximum at zero temperature, in odd-frequency states the maximum of $|\Delta E|$ is located near the upper critical temperature. Consequently, there is strong variation of all thermodynamic quantities with temperature just below $T_{c}$. Again, at lower temperatures these phenomena disappear.

The model that we have introduced is certainly simplified, as it ignores the possible proximity of other phases. However, we believe that odd-frequency density-wave order can result when a tendency towards ordinary evenfrequency density-wave order is frustrated by some competing interaction. The resolution of the competition will depend on the scales at which the various interactions act - essentially $\Omega_{c}$ in our model. In particular, one can imagine a scenario in which an ordinary even-frequency density wave is favorable at higher temperatures, but below a certain temperature, the system undergoes a transition into an odd-frequency state.

\section{ACKNOWLEDGMENTS}

We would like to thank E. Abrahams for discussions. C.N. is supported by the National Science Foundation under Grant No. DMR-9983544 and by the Alfred P. Sloan Foundation. E.P. is supported in part by the Department of Energy under grant DE-FG03-ER-40701.

[1] A frequency-independent order parameter may have Fermi pockets, but these are to be contrasted with the large true Fermi surface of a Fermi liquid. An oddfrequency ordered state has a Fermi surface of the latter variety.

[2] A. Balatsky and E. Abrahams, Phys. Rev. B 45, 13125 (1992).

[3] E. Abrahams, A. Balatsky, J. R. Schrieffer, and P. Allen, Phys. Rev. B 47, 513 (1993).

[4] E. Abrahams, A. Balatsky, D. J. Scalapino, and J. R. Schrieffer, Phys. Rev. B 52, 1271 (1995).

[5] P. Coleman, E. Miranda, and A. Tsvelik, Phys. Rev. Lett. 70, 2960 (1993).

[6] D. Belitz and T. R. Kirkpatrick, Phys. Rev. B 60, 3485 (1999) and references therein.

[7] A. Heid, Z. Phys. B 99, 15 (1995).

[8] O. Zachar, S. A. Kivelson, and V. J. Emery, Phys. Rev. Lett. 77, 1342 (1996). 
[9] P. Coleman, A. Georges, and A. Tsvelik, J. Phys.: Cond. Matt. 79, 345 (1997).

[10] S. Yip and A. J. Legget, unpublished.

[11] A. Balatsky and E. Abrahams, Phys. Rev. Lett. 74, 1004 (1995).

[12] Some care is required since both the kinetic and interaction terms in the mean-field action contain a single time-derivative. As a result, the commutations relations are modified. However, the resulting equations for $G$ and $F$ are of the same form as one would obtain by ignoring this fact and ignoring the time-derivative arising from the frequency dependence of the gap in (15), i.e., by making two compensating errors.

[13] The derivation of the "anomalous" last term in (26) is straightforward from the partition function using the path integral formalism. It is much more difficult, if at all possible, to explain its presence within classical thermodynamics. It is manifestly a quantum phenomenon.

[14] N. P. Ong, Physica C 235-240, 221 (1994), and references therein; A. W. Tyler et al., Phys. Rev. B 58, R10107 (1998).

[15] S. V. Kravchenko et al., Phys. Rev. B 51, 7038 (1995); P. T. Coleridge et al., Phys. Rev. B 56, R12764 (1997); S. J. Papadakis and M. Shayegan, Phys. Rev. B 57, R15068 (1998); Y. Hanein et al., Nature 400, 735 (1999); M. Y. Simmons and A. R. Hamilton, Nature 400, 715 (1999) 